\title{
Traditional Chinese Medicine Brucea Javanica Oil Enhances the Efficacy of Anlotinib in a Mouse Model of Liver-Metastasis of Small-cell Lung Cancer
}

\author{
SONG PENG ${ }^{1,2}$, WENHONG DONG ${ }^{1,2}$, QIANGQIANG CHU ${ }^{1,2}$, JIA MENG $^{1,2}$, \\ HAITAO YANG ${ }^{1,2}$, YINGYING DU ${ }^{3}$, YU SUN ${ }^{4,5}$ and ROBERT M. HOFFMAN ${ }^{4,5}$ \\ ${ }^{1}$ The Third Affiliated Hospital of Anhui Medical University, Hefei, P.R. China; \\ ${ }^{2}$ Hefei First People's Hospital, Hefei, P.R. China; \\ ${ }^{3}$ The First Affiliated Hospital of Anhui Medical University, Hefei, P.R. China; \\ ${ }^{4}$ AntiCancer Inc., San Diego, CA, U.S.A.; \\ ${ }^{5}$ Department of Surgery, UCSD, San Diego, CA, U.S.A.
}

\begin{abstract}
Background/Aim: Small-cell lung cancer (SCLC) is a recalcitrant disease with liver and other metastasis. The present study evaluated the efficacy of the traditional Chinese medicine Brucea javanica oil (BJO) combined with anlotinib, a multi-tyrosine kinase inhibitor with antiangiogenic activity, on a nude-mouse model of SCLC liver metastasis. Materials and Methods: The mouse model was established by injecting NCI-H446 cells $\left(1 \times 10^{6}\right)$ in Matrigel $(20 \mu l)$ into the upper liver lobe. All animals were randomized and assigned to three groups: Control $(n=8)$; anlotinib alone $(n=8 ; 3 \mathrm{mg} / \mathrm{kg}, q d \times 14+7$-day interval with two cycles, oral); anlotinib plus BJO $(n=8 ; 3 \mathrm{mg} / \mathrm{kg}$ anlotinib $q d \times 14+7-d a y$ interval with two cycles, orally; BJO: $1 \mathrm{~g} / \mathrm{kg}$, qdx6 weeks, orally). Body weight was determined every week. Six weeks after initial treatment, tumors were collected for analysis of angiogenesis using immunohistochemistry. Results: The combination of anlotinib and BJO significantly inhibited growth of SCLC liver metastases and angiogenesis more than anlotinib monotherapy ( $p=0.043)$. In addition, BJO alleviated bodyweight loss associated with anlotinib therapy, including general mouse condition. Conclusion: The results of the present study indicate that the combination of anlotinib with
\end{abstract}

This article is freely accessible online.

Correspondence to: Song Peng, The Third Affiliated Hospital of Anhui Medical University \& Hefei First People's Hospital, Hefei, P.R. China. Tel: +86 13956060263, e-mail: ps8491@ sina.com

Key Words: Brucea javanica oil, small-cell lung cancer, SCLC, nude mouse, liver metastasis, anlotinib, angiogenesis, combination therapy, efficacy.
BJO is promisingly active against liver metastases of SCLC, and has clinical potential.

Lung cancer is responsible for the greatest number of cancer deaths in the world $(1,2)$. There are two major types of lung cancer according to histopathological classification: nonsmall-cell lung cancer and small-cell lung cancer (SCLC). SCLC is an aggressive neuroendocrine malignancy with a high proliferative index, which accounts for $15 \%$ of all lung cancer $(3,4)$. However, because of its rapid progression and early metastasis (5), the 5-year survival rate of patients with SCLC is less than 5\%, lower than that for non-small-cell lung cancer (6). Although cytotoxic therapy has initial efficacy for SCLC, there is recurrence in most patients (7). Recurrent metastasis is recalcitrant, with only $5 \%$ survival (8).

Brucea javanica, a traditional Chinese medicine listed in the official Chinese Pharmacopoeia (9), is an evergreen plant shrub from the Simaroubaceae family that grows abundantly throughout Southern China and Southeast Asia (10). Oil from B. javanica (BJO) has been used to treat diarrhea (11), malaria (12), intestinal inflammation (13), and various types of cancer $(9,14-17)$.

Anlotinib is a small-molecule, multi-targeting tyrosine kinase inhibitor, which can inhibit vascular endothelial growth factor receptors 2, and 3, platelet-derived growth factor receptor, and stem cell-factor receptor (18). Based on the ALTER 1202 clinical trial, the China Food and Drug Administration approved anlotinib for use in third-line treatment in recurrent SCLC or SCLC with distant metastasis (19).

We previously reported that BJO enhanced gemcitabine efficacy in a mouse model of pancreatic cancer (20). In the present study, we combined BJO with anlotinib for the treatment of SCLC liver metastasis in a nude-mouse model. 


\section{Materials and Methods}

Cell culture. The human SCLC cell line NCI-H446 was purchased from the American Type Culture Collection (Vanassas, VA, USA). Cells were maintained in RPMI-1640 culture medium supplemented with $10 \%$ heat-inactivated fetal bovine serum and $1 \%$ penicillin, and cultured at $37^{\circ} \mathrm{C}$ in an incubator with $5 \% \mathrm{CO}_{2}$.

Animals. A total of 24 female athymic nude (nu/nu) mice, purchased from Cavens Inc. (Changzhou, Jiangsu, China), 5-6 weeks old, 20 2 g body weight, were used for the study. All animals were maintained in a high-efficiency particulate air-filtered environment at the animal research laboratory of Anhui medical university (Hefei, Anhui, PR China). Cages, food and bedding were autoclaved.

ExperimentaI agents. Anlotinib was purchased from Chia Tai Tianqing Pharmaceutical Group Co., Ltd (Lian Yungang, Jiangsu, PR China). Anlotinib was dissolved in dimethyl sulfoxide to prepare stock solution at a concentration of $16 \mathrm{mmol} / 1$, and stored at $-20^{\circ} \mathrm{C}$. Before treatment, the stock solution was diluted to $0.75 \mathrm{mg} / \mathrm{ml}$ using distilled water.

BJO capsules were purchased from Vanguard Pharmaceutical (Haimen, Jiangsu, PR China). BJO was dissolved in distilled water to a concentration of $250 \mathrm{mg} / \mathrm{ml}$, and stored at $4^{\circ} \mathrm{C}$.

Establishment of a nude mouse model of liver metastasis from SCLC. An SCLC liver-metastasis mouse model was established by injecting NCI-H446 cells $\left(1 \times 10^{6}\right)$ in Matrigel $(20 \mu \mathrm{l})(\mathrm{BD}$, Franklin Lakes, NJ, USA) into the upper liver lobe of nude mice. All surgical procedures were performed under $8 \times$ magnification in a highefficiency particulate air-filtered laminar flow hood. Animals were anesthetized by intramuscular injection of a ketamine mixture. The surgical area was sterilized using iodine and alcohol. A transverse incision of approximately $1 \mathrm{~cm}$ was made on the upper abdomen of the mouse using surgical scissors. The liver was exposed, and NCIH446 cells $(20 \mu \mathrm{l})$ were injected into the liver lobe. Bleeding at the injection site was stopped by cotton swab pressure. The incision was closed with 5-0 surgical sutures.

Treatment. Treatment was started 1 week after NCI-H446-cell injection. All animals were randomized and assigned to three groups of eight animals each: Control; anlotinib ( $3 \mathrm{mg} / \mathrm{kg}, q d \times 14+7$-day interval for two cycles, oral administration by gavage) (21); anlotinib as above plus BJO ( $1 \mathrm{~g} / \mathrm{kg}, q d \times 6$ weeks, gavage) (20). Body weight was determined every week using an electrical scale throughout the treatment period. The study was terminated 6 weeks after the initiation of treatment. All animals were euthanized and examined at necropsy. The abdominal cavity was opened to expose the liver. Tumor volume was estimated by measuring the perpendicular minor dimension (W) and major dimension (L). Approximate tumor volume was calculated with the formula $\left(\mathrm{W}^{2} \times \mathrm{L}\right) / 2$. The tumors were resected and tumor weight was determined using an electronic scale. The tumor growth inhibition (TGI) in each group was calculated with the formula: TGI $(\%)=(1-$ mean tumor weight of the treated group/mean tumor weight of the control group) $\times 100 \%$. The tumor samples were stored in $10 \%$ formalin for histopathological examination.

Immunohistochemistry. The expression of CD34 was detected with an immunohistochemical assay to compare the extent of tumor microvessel area between each group. Formaldehyde-fixed tumors were embedded in paraffin and the sections $(4 \mu \mathrm{m})$ were obtained using a microtome (RM2125; Leica, Wetzlar, Germany). Sections were deparaffinized after hydration, and antigen retrieval buffer was used for 30 minutes. After endogenous peroxidase was quenched with $0.1 \%$ hydrogen peroxide for 15 minutes, the samples were blocked using serum-free protein and incubated with primary rabbit monoclonal against CD34 (ab110643; Abcam, Cambridge, MA, USA) overnight. Following washing with phosphate buffer saline, sections were incubated with a sheep anti-rabbit IgG secondary antibody (ab172730; Abcam), followed by incubation with 3\% peroxidase (Invitrogen, Waltham, MA, USA) and a solution of $0.1 \%$ 3, 3-diaminobenzidine and $0.02 \% \quad \mathrm{H}_{2} \mathrm{O}_{2}$. The sections were examined and positive cells were recognized by their brown-stained appearance. Expression levels were quantified by the average optical density of the positive cells in five fields/sample with ImagePro Plus 6.0 software (Media Cybernetics, Silver Springs, MD, USA) (22).Typical images were captured using a microscope (BX53F; Olympus Corp, Tokyo, Japan) with its software.

Statistical analysis. All data were analyzed with GraphPad Prism 8.2.1 software (GraphPad Software Inc., La Jolla, CA, USA). All data are represented as the mean \pm standard deviation. One-way analysis of variance followed by Tukey's correction were used to determine statistical differences in the mean tumor volume, tumor weight and body weight among the experimental groups. Values of $p \leq 0.05$ were considered statistically significant.

\section{Results}

BJO enhances anlotinib efficacy against liver metastasis from SCLC. All treatment groups resulted in statistically significantly smaller tumor volumes (Figure 1) and weights compared to the vehicle-treated control at the end of treatment $(p<0.05)$. The addition of BJO significantly increased efficacy compared to the anlotinib monotherapy, both for tumor volume $(p=0.024)$ and tumor weight $(p=0.037)$ (Table I). The TGI value in the combination-treated group was $53.44 \%$ compared to that of anlotinib monotherapy of $21.82 \%$.

BJO reduced anlotinib-induced weight loss in the nudemouse model of SCLC liver metastasis. Mice treated with the combination of anlotinib and BJO demonstrated significantly lower body-weight loss compared to those on anlotinib monotherapy during the entire treatment period $(p=0.043)$ (Figure 2). The greater activity and smoother skin of the combination-treated mice indicated that BJO improved their general condition.

BJO enhanced the anti-angiogenic effect of amlotinib. Although anlotinib monotherapy significantly inhibited growth of tumor microvessels compared to the vehicle control group ( $p=0.034)$, the combination of anlotinib and BJO showed significantly higher anti-angiogenic efficacy compared to mice of vehicle control $(p=0.001)$ and anlotinib monotherapy ( $p=0.023$ ) group (Figure 3 ). 


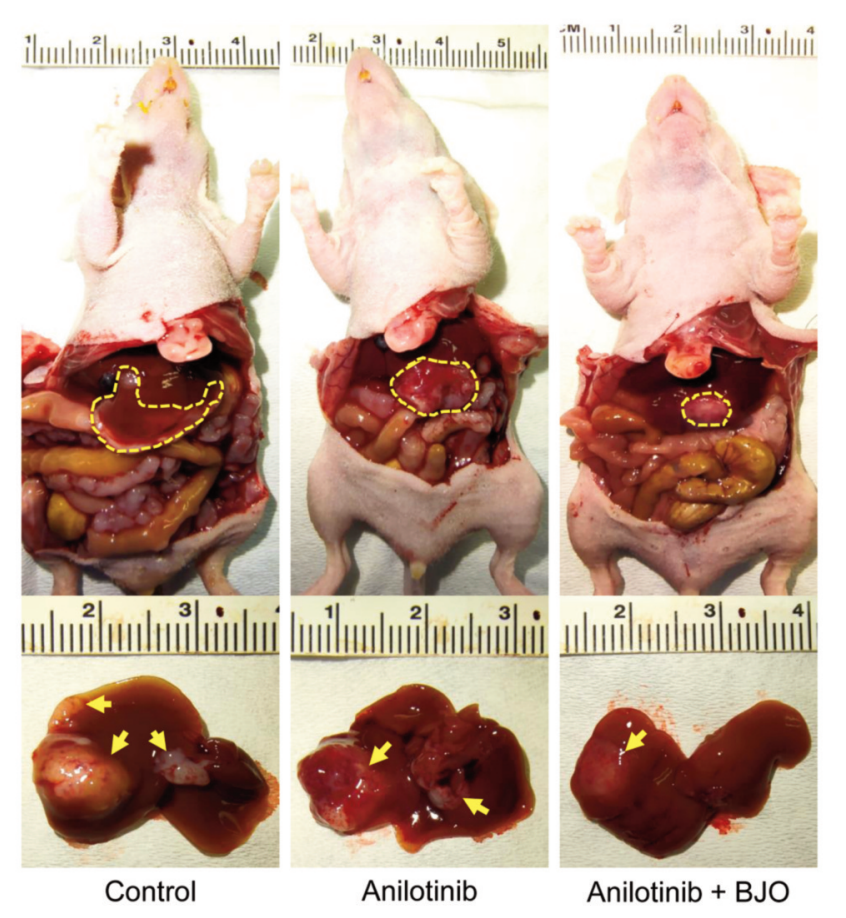

Figure 1. Inhibitory effect of the combination of Brucea javanica oil and anlotinib on small cell lung cancer liver metastases in a nude-mouse model. Images of liver metastasis tumor in each group at necropsy, liver metastases are shown with yellow arrows and dashed line.

Table I. Efficacy of treatment on final tumor volume and weight in the nude-mouse model of liver metastasis of small-cell lung cancer.

\begin{tabular}{lcccc}
\hline Group & $\begin{array}{c}\text { Tumor volume } \\
\left(\mathrm{mm}^{3}\right) \\
\text { Mean } \pm \text { SD }\end{array}$ & $p$-Value & $\begin{array}{c}\text { Tumor weight } \\
(\mathrm{g}) \\
\text { Mean } \pm \text { SD }\end{array}$ & $p$-Value \\
\hline Control & $396.9 \pm 70.8$ & & $2.87 \pm 0.65$ & \\
Anlotinib & $302.5 \pm 57.5$ & $\mathbf{0 . 0 2 5}^{*}$ & $2.24 \pm 0.44$ & $\mathbf{0 . 2 3 1}^{*}$ \\
Anlotinib+BJO & $180.8 \pm 48.8$ & $\mathbf{0 . 0 0 1}^{*}$ & $1.34 \pm 0.45$ & $\mathbf{0 . 0 0 8}^{*}$ \\
& & $\mathbf{0 . 0 2 4}^{\#}$ & & $\mathbf{0 . 0 3 7}^{\#}$ \\
\hline
\end{tabular}

Bold values indicate statistically significant difference. Versus * Control, \#anlotinib-treated.

\section{Discussion}

The results of the present study indicate that the traditional Chinese medicine BJO both enhanced the efficacy of anlotinib on the growth of the SCLC liver metastases and prevented toxic body weight loss due therapy with anlotinib. SCLC is a recalcitrant disease in need of effective therapy (23). Although first-line chemotherapy is often effective for SCLC, the effect is temporary and there is almost always recurrence which leads to the patient's death. Liver

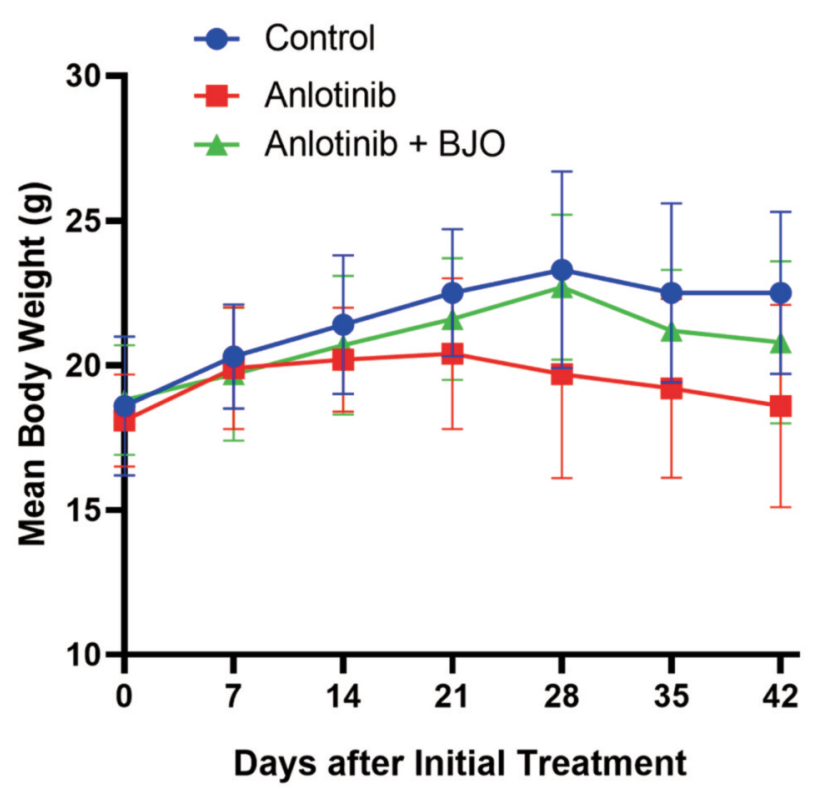

Figure 2. Effect of Brucea javanica oil on mouse body weight during the treatment period. Data are presented as the mean \pm standard deviation. *Significantly different from anlotinib monotherapy at $p<0.05$.

metastasis, along with brain metastasis, are the major causes of death in patients with $\operatorname{SCLC}(24,25)$. Although anlotinib has been approved for third-line therapy, in China it has shown only moderate clinical efficacy (18), and also showed moderate efficacy in the present study. In addition, drugrelated adverse reactions are often found in patients, including fatigue (17.7\%), weight loss $(17.7 \%)$, and hematological toxicity (13.9\%) (26). The dose of anlotinib used in this study had significant toxicity, as shown by mouse body-weight loss.

Our previous study showed that BJO enhanced gemcitabine efficacy and increased survival in a patientderived orthotopic xenograft mouse model of pancreatic cancer (20). In the present study, we first showed BJO can be effectively combined with anlotinib in a nude-mouse model of SCLC liver-metastasis, where in combination with anlotinib it inhibited both liver metastasis growth and tumor angiogenesis in the liver. BJO also alleviated the weight loss due to anlotinib, and improved mouse body condition.

In brief, BJO both enhanced anlotinib efficacy while reducing toxicity, indicating the future clinical potential of the combination of BJO and anlotinib for SCLC livermetastasis, a recurrently recalcitrant disease.

\section{Conflicts of Interest}

All the Authors declare no conflicts of interest in this study. 

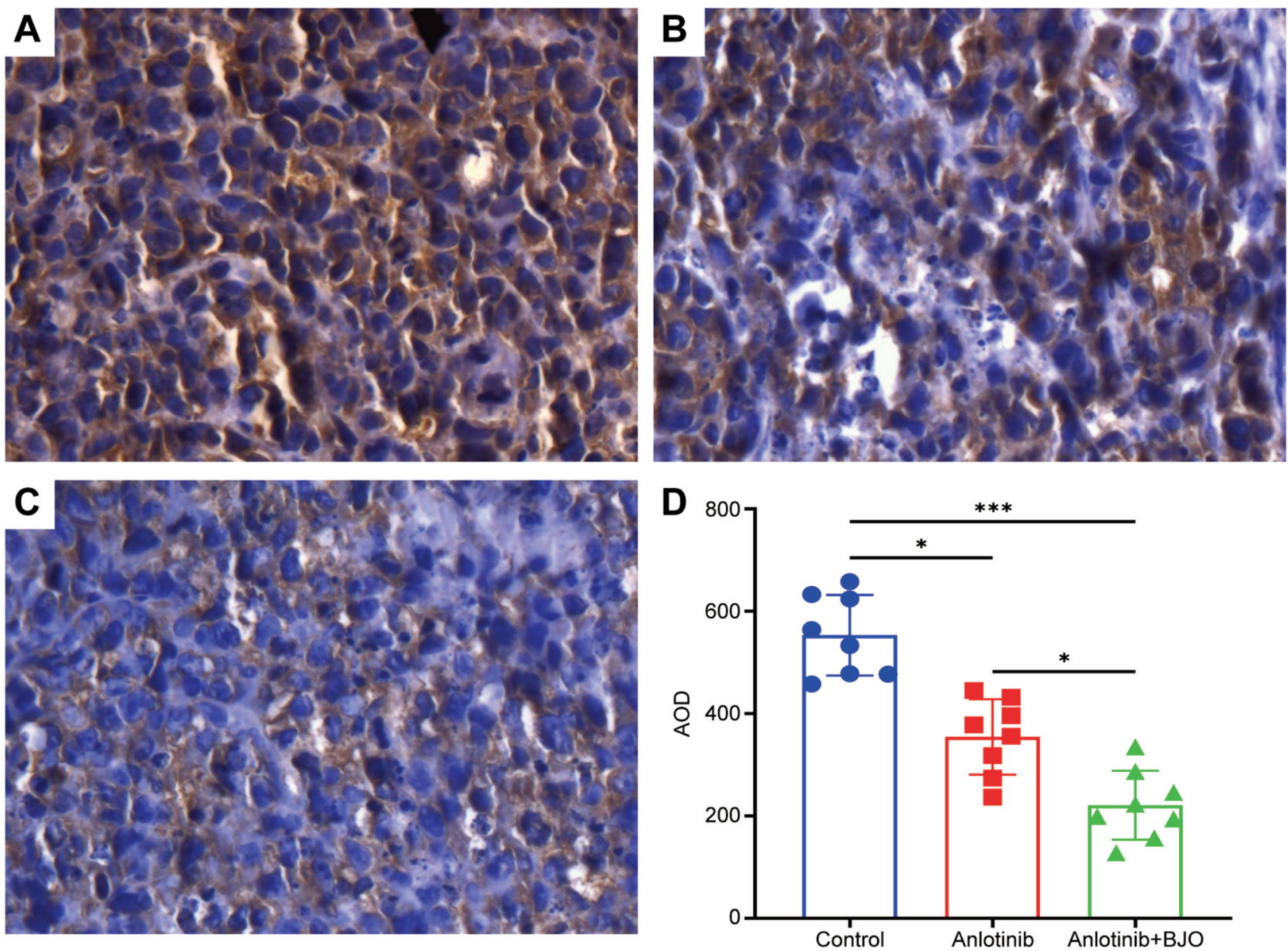

Figure 3. Anti-angiogenic efficacy of the combination of Brucea javanica oil and anlotinib. Representative images are shown of CD34 (brown)stained tumor reflecting angiogenesis in each group (200x magnification). A: Control; B: anlotinib monotherapy; C: BJO combined with anlotinib. D: Quantification of tumor microvessel area was quantified by the average optical density (AOD) in each group. Data are presented as the mean \pm standard deviation. Significantly different at $* p<0.05$ and $* * * p<0.001$.

\section{Authors' Contributions}

SP designed the study. SP, WD, QC, JM performed the experiments. YS analyzed the data. SP and HY drafted the article. RMH revised the article and rewrote the Discussion. JH administrated and supervised the study.

\section{Acknowledgements}

This study was supported by Key Research and Development Project of Anhui Province (No. 1704a0802163) and Hefei Key Medical Discipline Construction Fund (No. 2019-160).

\section{References}

1 Chen W, Zheng R, Baade PD, Zhang S, Zeng H, Bray F, Jemal A, $\mathrm{Yu}$ XQ and He J: Cancer statistics in China, 2015. CA Cancer J Clin 66(2): 115-132, 2016. PMID: 26808342. DOI: 10.3322/caac.21338
2 Yu G, Shen Y, Xu X and Zhong F: Anlotinib for refractory advanced non-small-cell lung cancer: A systematic review and meta-analysis. PLoS One 15(11): e0242982, 2020. PMID: 33253313. DOI: 10.1371/journal.pone.0242982

3 Govindan R, Page N, Morgensztern D, Read W, Tierney R, Vlahiotis A, Spitznagel EL and Piccirillo J: Changing epidemiology of small-cell lung cancer in the United States over the last 30 years: analysis of the surveillance, epidemiologic, and end results database. J Clin Oncol 24(28): 4539-4544, 2006. PMID: 17008692. DOI: 10.1200/JCO.2005.04.4859

4 Siegel RL, Miller KD and Jemal A: Cancer statistics, 2019. CA Cancer J Clin 69(1): 7-34, 2019. PMID: 30620402. DOI: 10.3322/caac. 21551

5 Mishima S, Nozaki Y, Mikami S, Kihira E, Iikura M, Koketsu R, Sugiyama H, Masuda T, Kaname H, Egami Y, Nakayama T, Hasuo K, Nakamura H, Igari T, Watanabe K, Nagata N, Sakurai T, Yokoi C, Kobayakawa M, Kojima Y, Akiyama J, Imamura M, Masaki N and Yanase M: Diffuse liver metastasis of small-cell lung cancer presenting as acute liver failure and diagnosed by 
transjugular liver biopsy: A rare case in whom nodular lesions were detected by enhanced CT examination. Case Rep Gastroenterol 9(1): 81-87, 2015. PMID: 25969674. DOI: 10.1159/ 000381140

6 Rodriguez E and Lilenbaum RC: Small cell lung cancer: Past, present, and future. Curr Oncol Rep 12(5): 327-334, 2010 PMID: 20632219. DOI: 10.1007/s11912-010-0120-5

7 Chang KJ, Yin JZ, Huang H, Li B and Yang MH: Arsenic trioxide inhibits the growth of cancer stem cells derived from small cell lung cancer by downregulating stem cell-maintenance factors and inducing apoptosis via the Hedgehog signaling blockade. Transl Lung Cancer Res 9(4): 1379-1396, 2020. PMID: 32953511. DOI: $10.21037 /$ tlcr-20-467

8 Ou SH, Ziogas A and Zell JA: Prognostic factors for survival in extensive stage small cell lung cancer (ED-SCLC): the importance of smoking history, socioeconomic and marital statuses, and ethnicity. J Thorac Oncol 4(1): 37-43, 2009. PMID: 19096304. DOI: 10.1097/JTO.0b013e31819140fb

9 Yan Z, Guo GF and Zhang B: Research of Brucea javanica against cancer. Chin J Integr Med 23(2): 153-160, 2017. PMID: 27041332. DOI: $10.1007 / \mathrm{s} 11655-016-2501-6$

10 Zhao L, Li C, Zhang Y, Wen Q and Ren D: Phytochemical and biological activities of an anticancer plant medicine: Brucea javanica. Anticancer Agents Med Chem 14(3): 440-458, 2014. PMID: 24066797. DOI: 10.2174/18715206113136660336

11 Tangpu V and Yadav AK: Antidiarrhoeal activity of Rhus javanica ripen fruit extract in albino mice. Fitoterapia 75(1): 3944, 2004. PMID: 14693218. DOI: 10.1016/j.fitote.2003.08.015

12 O'Neill MJ, Bray DH, Boardman P, Chan KL, Phillipson JD, Warhurst DC and Peters W: Plants as sources of antimalarial drugs, Part 4: Activity of Brucea javanica fruits against chloroquine-resistant Plasmodium falciparum in vitro and against Plasmodium berghei in vivo. J Nat Prod 50(1): 41-48, 1987. PMID: 3298551. DOI: 10.1021/np50049a007

13 Huang YF, Zhou JT, Qu C, Dou YX, Huang QH, Lin ZX, Xian YF, Xie JH, Xie YL, Lai XP and Su ZR: Anti-inflammatory effects of Brucea javanica oil emulsion by suppressing NF-кB activation on dextran sulfate sodium-induced ulcerative colitis in mice. J Ethnopharmacol 198: 389-398, 2017. PMID: 28119098. DOI: 10.1016/j.jep.2017.01.042

14 Yoon BK, Lim ZY, Jeon WY, Cho NJ, Kim JH and Jackman JA: Medicinal activities and nanomedicine delivery strategies for Brucea javanica Oil and Its Molecular Components. Molecules 25(22): 5414, 2020. PMID: 33228061. DOI: 10.3390/ molecules 25225414

15 Chen X, Li S, Li D, Li M, Su Z, Lai X, Zhou C, Chen S, Li S, Yang X, Su J and Zhang Y: Ethanol extract of Brucea javanica seed inhibit triple-negative breast cancer by restraining autophagy via PI3K/Akt/mTOR pathway. Front Pharmacol 11: 606, 2020. PMID: 32411003. DOI: 10.3389/fphar.2020.00606

16 Yan Z, Zhang B, Huang Y, Qiu H, Chen P and Guo GF: Involvement of autophagy inhibition in Brucea javanica oil emulsion-induced colon cancer cell death. Oncol Lett 9(3): 1425-1431, 2015. PMID: 25663926. DOI: 10.3892/ol.2015.2875
17 Wu JR, Liu SY, Zhu JL, Zhang D and Wang KH: Efficacy of Brucea javanica oil emulsion injection combined with the chemotherapy for treating gastric cancer: A systematic review and meta-analysis. Evid Based Complement Alternat Med 2018: 6350782, 2018. PMID: 29853964. DOI: 10.1155/2018/6350782

18 Han B, Li K, Zhao Y, Li B, Cheng Y, Zhou J, Lu Y, Shi Y, Wang Z, Jiang L, Luo Y, Zhang Y, Huang C, Li Q and Wu G: Anlotinib as a third-line therapy in patients with refractory advanced nonsmall-cell lung cancer: A multicentre, randomised phase II trial (ALTER0302). Br J Cancer 118(5): 654-661, 2018. PMID: 29438373. DOI: $10.1038 /$ bjc.2017.478

19 Yang S, Zhang Z and Wang Q: Emerging therapies for small cell lung cancer. J Hematol Oncol 12(1): 47, 2019. PMID: 31046803. DOI: $10.1186 / \mathrm{s} 13045-019-0736-3$

20 Yang H, Tong Z, Shen L, Sun YU, Hoffman RM and Huang J: Brucea javanica increases survival and enhances gemcitabine efficacy in a Patient-derived Orthotopic Xenograft (PDOX) Mouse Model of Pancreatic Cancer. Anticancer Res 40(9): 49694978, 2020. PMID: 32878785. DOI: 10.21873/anticanres.14500

21 Qin T, Liu Z, Wang J, Xia J, Liu S, Jia Y, Liu H and Li K: Anlotinib suppresses lymphangiogenesis and lymphatic metastasis in lung adenocarcinoma through a process potentially involving VEGFR-3 signaling. Cancer Biol Med 17(3): 753-767, 2020. PMID: 32944404. DOI: 10.20892/j.issn.2095-3941. 2020.0024

22 Yang H, Ding R, Tong Z, Huang J, Shen L, Sun YU, Liao J, Yang Z, Hoffman RM, Wang C and Meng X: siRNA targeting of MDR1 reverses multidrug resistance in a nude mouse model of Doxorubicin-resistant human hepatocellular carcinoma. Anticancer Res 36(6): 2675-2682, 2016. PMID: 27272776.

23 Teicher BA: Perspective: Opportunities in recalcitrant, rare and neglected tumors. Oncol Rep 30(3): 1030-1034, 2013. PMID: 23820887. DOI: 10.3892/or.2013.2581

24 Ellis LM and Hicklin DJ: VEGF-targeted therapy: Mechanisms of anti-tumour activity. Nat Rev Cancer 8(8): 579-591, 2008. PMID: 18596824. DOI: $10.1038 / \mathrm{nrc} 2403$

25 Fontanini G, Faviana P, Lucchi M, Boldrini L, Mussi A, Camacci T, Mariani MA, Angeletti CA, Basolo F and Pingitore R: A high vascular count and overexpression of vascular endothelial growth factor are associated with unfavourable prognosis in operated small cell lung carcinoma. Br J Cancer 86(4): 558-563, 2002. PMID: 11870537. DOI: 10.1038/sj.bjc. 6600130

26 Song PF, Xu N and Li Q: Efficacy and safety of Anlotinib for elderly patients with previously treated extensive-stage SCLC and the prognostic significance of common adverse reactions. Cancer Manag Res 12: 11133-11143, 2020. PMID: 33173346. DOI: $10.2147 /$ CMAR.S275624

Received February 7, 2021

Revised March 3, 2021

Accepted March 4, 2021 